\title{
GLOBAL APPROXIMATION BY MODIFIED BASKAKOV TYPE OPERATORS
}

\author{
ViJAY GuPtA
}

Abstract

In the present paper, we prove a global direct theorem for the modified Baskakov type operators in terms of so called DitzianTotik modulus of smoothness.

\section{Introduction}

Motivated by the integral modification of Bernstein polynomials by Durrmeyer [3], Sahai and Prasad [6] first defined and studied modified Baskakov operators. Sinha et al. [7] improved and corrected the results of [6]. Recently the author [4], introduced another modification of Baskakov operators by taking the weight function of Beta operators on $L_{1}[0, \infty)$ as

$$
\left(B_{n} f\right)(x)=\sum_{k=0}^{\infty} p_{n, k}(x) \int_{0}^{\infty} b_{n, k}(t) f(t) d t, \quad x \in[0, \infty)
$$

where

$$
p_{n, k}(x)=\left(\begin{array}{c}
n+k-1 \\
k
\end{array}\right) x^{k}(1+x)^{-n-k}
$$

and

$$
b_{n, k}(t)=[B(k+1, n)]^{-1} t^{k}(1+t)^{-n-k-1},
$$

$B(k+1, n)$ being the Beta function given by $k !(n-1) ! /(n+k)$ !.

In [4], the author has obtained only local direct theorems in simultaneous approximation, as the operators defined by (1.1) give better approximation than the earlier integral modification of Baskakov operators

Research supported by Council of Scientific and Industrial Research, India under award no. 9/143(163)/91-EMR-1. 
studied in [5], $[\mathbf{6}]$ and $[\mathbf{7}]$ etc., this motivated us to extend the results of $[4]$ to the whole interval $[0, \infty)$ and we study a global result for the operators (1.1).

By $\mathcal{L}_{1}^{r}[0, \infty)$, we denote the class of functions $g$ given by

$$
\begin{aligned}
& \mathcal{L}_{1}^{r}[0, \infty):=\left\{g: g^{(r)} \in L_{1}[0, a] \text { for every } a \in(0, \infty)\right. \text { and } \\
& \left.\left|g^{(r)}(t)\right| \leq M(1+t)^{m}, M \text { and } m \text { are constants depending on } g\right\} .
\end{aligned}
$$

We may remark that $L_{p}^{r}[0, \infty)$ is not contained in $\mathcal{L}_{1}^{r}[0, \infty)$.

Following [2], the modulus of smoothness of $f$ is given by

$$
\omega_{\phi}^{2}(f, t)_{p}=\sup _{0<h \leq t}\left\|\Delta_{h \phi}^{2} f\right\|_{p}, \phi(x)=\sqrt{x(1+x)}
$$

where

$$
\Delta_{h}^{2} f(x)= \begin{cases}f(x-h)-2 f(x)+f(x+h), & \text { if }[x-h, x+h] \subset[0, \infty) \\ 0, & \text { otherwise }\end{cases}
$$

This modulus of smoothness is equivalent to the modified $k$-functional (see e.g. [2]) given by

$$
\bar{K}_{\phi}^{2}\left(f, t^{2}\right)_{p}=\inf \left\{\|f-g\|_{p}+t^{2}\left\|\phi^{2} g^{\prime \prime}\right\|_{p}+t^{4}\left\|g^{\prime \prime}\right\|_{p} ; g \in \bar{W}_{p}^{2}(\phi,[0, \infty))\right\}
$$

where

$$
\bar{W}_{p}^{2}(\phi,[0, \infty))=\left\{g \in L_{p}[0, \infty): g^{\prime} \in A C_{\mathrm{loc}}[0, \infty) ; \phi^{2} g^{\prime \prime} \in L_{p}[0, \infty)\right\} .
$$

In [4] the author was not able to obtain global results. In the present paper, we prove a global direct theorem in simultaneous approximation for the operators $\left(B_{n} f\right)(x)$ defined by (1.1) in terms of Ditzian-Totik modulus of second order.

Throughout the paper we denote by $C$ the positive constants not necessarily the same at each occurrence.

\section{Auxiliary results}

In this section, we shall give certain definitions and lemmas which will be used in the sequel.

For every $n \in N$ and $n>(r+1)$ we have

$$
\begin{array}{ll}
\sum_{k=0}^{\infty} p_{n, k}(x)=1, & \int_{0}^{\infty} b_{n, k}(t) d t=1 \\
\frac{k}{n} p_{n, k}(x)=x p_{n+1, k-1}(x), & \int_{0}^{\infty} t b_{n-r, k+r}(t) d t=\frac{k+r+1}{n-r-1} .
\end{array}
$$


Lemma 2.1 [4]. Let $m, r \in N_{0}$, we define

$$
T_{r, n, m}(x)=\sum_{k=0}^{\infty} p_{n+r, k}(x) \int_{0}^{\infty} b_{n-r, k+r}(t)(t-x)^{m} d t
$$

then

$$
\begin{aligned}
& T_{r, n, 0}(x)=1, T_{r, n, 1}(x)=\frac{1+r+x(1+2 r)}{(n-r-1)} \\
& T_{r, n, 2}(x)=\frac{2\left(2 r^{2}+4 r+n+1\right) x^{2}+2\left(2 r^{2}+5 r+2+n\right) x+\left(r^{2}+3 r+2\right)}{(n-r-1)(n-r-2)},
\end{aligned}
$$

and there holds the recurrence relation:

$$
\begin{aligned}
& (n-m-r-1) T_{r, n, m+1}(x)=\phi^{2}(x)\left[T_{r, n, m}^{(1)}(x)+2 m T_{r, n, m-1}(x)\right] \\
& +[(m+r+1)(1+2 x)-x] T_{r, n, m}(x), \quad n>m+r+1 .
\end{aligned}
$$

Consequently for each $x \in[0, \infty), T_{r, n, m}(x)=0\left(n^{-[(m+1) / 2]}\right),[\alpha] d e-$ notes the integral part of $\alpha$.

The proof of this lemma easily follows along the lines of $[\mathbf{6}],[\mathbf{7}]$ using $\phi^{2}(x) p_{n, k}^{\prime}(x)=(k-n x) p_{n, k}(x)$ and $\phi^{2}(t) b_{n, k}^{\prime}(t)=[k-(n+1) t] b_{n, k}(t)$.

From the above lemma, we have

$$
\begin{aligned}
T_{r, n, 2 m}(x) & =\sum_{i=0}^{m} q_{i, m, n}(x)\left[\frac{\phi^{2}(x)}{n}\right]^{m-i} n^{-2 i} \\
T_{r, n, 2 m+1}(x) & =(1+2 x) \sum_{i=0}^{m} s_{i, m, n}(x)\left[\frac{\phi^{2}(x)}{n}\right]^{m-i} n^{-2 i-1}
\end{aligned}
$$

where $q_{i, m, n}(x)$ and $s_{i, m, n}(x)$ are polynomials in $x$ of fixed degree with coefficients that are bounded uniformly for all $n$.

Lemma 2.2. If $f \in L_{p}^{r}[0, \infty) \cup \mathcal{L}_{1}^{r}[0, \infty), 1 \leq p \leq \infty, n>r(1+m)$ and $x \in[0, \infty)$, then

$$
\left(B_{n} f\right)^{(r)}(x)=\alpha(n, r) \sum_{k=0}^{\infty} p_{n+r, k}(x) \int_{0}^{\infty} b_{n-r, k+r}(t) f^{(r)}(t) d t
$$


where

$$
\alpha(n, r)=\frac{(n+r-1) !(n-r-1) !}{((n-1) !)^{2}}=\prod_{\ell=0}^{r-1} \frac{n+\ell}{n-(\ell+1)} .
$$

Proof: By using Leibnitz theorem, we have

$$
\begin{aligned}
\left(B_{n} f\right)^{(r)}(x)= & \sum_{i=0}^{r} \sum_{k=i}^{\infty}\left(\begin{array}{l}
r \\
i
\end{array}\right) \frac{(n+k+r-i-1) !}{(n-1) !(k-i) !} \\
& \times(-1)^{r-i} x^{k-i}(1+x)^{-n-k-r+i} \\
& \times \int_{0}^{\infty} b_{n, k}(t) f(t) d t \\
= & \frac{(n+r-1) !}{(n-1) !} \sum_{k=0}^{\infty} p_{n+r, k}(x) \\
& \times \int_{0}^{\infty} \sum_{i=0}^{r}(-1)^{r-i}\left(\begin{array}{l}
r \\
i
\end{array}\right) b_{n, k+i}(t) f(t) d t
\end{aligned}
$$

Again, by the use of Leibnitz theorem, we have

$$
b_{n-r, k+r}^{(r)}(t)=\frac{(n-1) !}{(n-r-1) !} \sum_{i=0}^{r}(-1)^{i}\left(\begin{array}{l}
r \\
i
\end{array}\right) b_{n, k+i}(t) .
$$

Hence,

$$
\begin{gathered}
\left(B_{n} f\right)^{(r)}(x)=\frac{(n+r-1) !(n-r-1) !}{((n-1) !)^{2}} \\
\sum_{k=0}^{\infty} p_{n+r, k}(x) \int_{0}^{\infty}(-1)^{r} b_{n-r, k+r}^{(r)}(t) f(t) d t .
\end{gathered}
$$

On integrating $r$ times by parts, we get the required result.

We see that the operators defined in $(2.3)$ by $B_{n}^{(r)} f:=\left(B_{n} f\right)^{(r)}, f \in$ $L_{p}^{r}[0, \infty) \cup \mathcal{L}_{1}[0, \infty)$ are not positive. To make the operators positive we introduce the operator

$$
B_{n, r} f \equiv D^{r} B_{n} I^{r} f, \quad f \in L_{p}[0, \infty) \cup \mathcal{L}_{1}[0, \infty),
$$

where $D$ and $I$ are differentiation and integration operators respectively. Therefore we define the operator by

$$
\left(B_{n, r} f\right)(x)=\alpha(n, r) \sum_{k=0}^{\infty} p_{n+r, k}(x) \int_{0}^{\infty} b_{n-r, k+r}(t) f(t) d t
$$


$f \in L_{p}[0, \infty) \cup \mathcal{L}_{1}[0, \infty), n>r(1+m)$.

The operators $B_{n, r}$ are positive and the estimation $\left\|\left(B_{n} f\right)^{(r)}-f^{(r)}\right\|_{p}$. $f \in L_{p}^{r}[0, \infty)$ is equivalent to $\left\|B_{n, r} f-f\right\|_{p}, f \in L_{p}[0, \infty)$.

Using (2.1), we can easily prove that for $n>(r+1),\left\|B_{n, r} f\right\|_{1} \leq$ $C\|f\|_{1}$, for $f \in L_{1}[0, \infty)$ and $\left\|B_{n, r} f\right\| \leq C\|f\|_{\infty}$ for $f \in L_{\infty}[0, \infty)$. Making use of Riesz-Thorin theorem, we get

$$
\left\|B_{n, r} f\right\|_{p} \leq C\|f\|_{p}, f \in L_{p}[0, \infty), 1 \leq p \leq \infty, n>(r+1)
$$

Corollary 2.3. For every $m \in N_{0}, n>(r+2 m+1)$ and $x \in[0, \infty)$ we have

$$
\begin{aligned}
\left|B_{n, r}\left((t-x)^{2 m}, x\right)\right| & \leq C n^{-m}\left(\phi^{2}(x)+n^{-1}\right)^{m} \\
\left|B_{n, r}\left((t-x)^{2 m+1}, x\right)\right| & \leq C(1+2 x) n^{-m-1}\left(\phi^{2}(x)+n^{-1}\right)^{m}
\end{aligned}
$$

where the constant $C$ is independent of $n$. For fixed $x \in[0, \infty)$ we obtain

$$
\left|B_{n, r}\left((t-x)^{m}, x\right)\right|=0\left(n^{-[(m+1) / 2]}\right), \quad n \rightarrow \infty .
$$

Proof: Since $B_{n, r}\left((t-x)^{m}, x\right)=\alpha(n, r) T_{r, n, m}(x)$ the estimate $(2.5)$ follows from (2.2) along the lines of [5], (2.6) immediately follows from $(2.5)$.

Lemma 2.4. Let $t \in[0, \infty)$ and $n>(r+m)$ then

$$
B_{n, r}\left((1+t)^{-m}, x\right) \leq C(1+x)^{-m}, \quad x \in[0, \infty)
$$

where the constant $C$ is independent of $n$.

Proof: It is easily verified that

$$
(1+t)^{-m} b_{n-r, k+r}(t)=\prod_{\ell=0}^{m-1} \frac{n-r+\ell}{n+\ell+k+1} b_{n-r+m, k+r}(t)
$$

and

$$
p_{n+r, k}(x)=(1+x)^{-m} \prod_{\ell=1}^{m} \frac{n+r-\ell+k}{n+r-\ell} p_{n+r-m, k}(x) .
$$


Making use of these two identities and (2.1) we get

$$
\begin{aligned}
B_{n, r}\left((1+t)^{-m}, x\right)= & \alpha(n, r) \sum_{k=0}^{\infty} p_{n+r, k}(x) \int_{0}^{\infty} b_{n-r, k+r}(t)(1+t)^{-m} d t \\
= & \alpha(n, r) \sum_{k=0}^{\infty} p_{n+r, k}(x) \prod_{\ell=0}^{m-1} \frac{n-r+\ell}{n+\ell+k+1} \\
& \times \int_{0}^{\infty} b_{n-r+m, k+r}(t) d t \\
= & \alpha(n, r) \sum_{k=0}^{\infty}(1+x)^{-m} p_{n+r-m, k}(x) \prod_{\ell=1}^{m} \frac{(n+r-\ell+k)}{(n+r-\ell)} \\
& \times \prod_{\ell=0}^{m-1} \frac{n+r-\ell}{n+\ell+k+1} \\
\leq & C(1+x)^{-m} \sum_{k=0}^{\infty} p_{n+r-m, k}(x) \\
= & C(1+x)^{-m} .
\end{aligned}
$$

For the two monomials $e_{0}, e_{1}$ and $x \in[0, \infty), n \rightarrow \infty$ we obtain by direct computation

$$
\begin{aligned}
& B_{n, r}\left(e_{0}, x\right)=1+0\left(n^{-1}\right) \\
& B_{n, r}\left(e_{1}, x\right)=x\left(1+0\left(n^{-1}\right)\right) .
\end{aligned}
$$

Lemma 2.5. For $H_{n}(u)$ given by

$$
H_{n}(u)=\left\{\int_{0}^{\infty} \int_{0}^{u}-\int_{0}^{u} \int_{0}^{\infty}\right\} \sum_{k=0}^{\infty} p_{n+r, k}(x) b_{n-r, k+r}(t)(u-t) d t d x
$$

we have $H_{n}(u) \leq C n^{-1} \phi^{2}(u)$, where $C$ is independent of $n$ and $u$.

The proof of the above lemma easily follows by using (2.1) along the lines of [1, Lemma 5.2].

\section{Direct result}

Theorem 3.1. Suppose $f \in L_{p}[0, \infty), 1 \leq p<\infty, n>(r+5)$ then we have

$$
\left\|B_{n, r} f-f\right\|_{p} \leq C\left\{\omega_{\phi}^{2}\left(f, n^{-1 / 2}\right)+n^{-1}\|f\|_{p}\right\}
$$


where the constant $C$ is independent of $n$.

Proof: By Taylor's expansion of $g$, we have

$$
g(t)=g(x)+(t-x) g^{\prime}(x)+\int_{x}^{t}(t-u) g^{\prime \prime}(u) d u .
$$

Next, since $B_{n, r}(f, x)$ are uniformly bounded operators so for every $g \in$ $\bar{W}_{p}^{2}(\phi,[0, \infty))$, we have

$$
\left\|B_{n, r} f-f\right\|_{p} \leq C\|f-g\|_{p}+\left\|B_{n, r} g-g\right\|_{p} .
$$

Using (2.5), (2.8) and (3.1) and following [2], we obtain

$$
\begin{aligned}
\left\|B_{n, r} g-g\right\|_{p} \leq & C\left\{\|g\|_{p}+\left\|g^{\prime}\right\|_{L_{p}[0,1]}\right\}+\left\|(1+2 x) g^{\prime}\right\|_{L_{p}[1, \infty)} \\
& +\left\|B_{n, r}(R(g, t, x), x)\right\|_{p} \\
\leq & C n^{-1}\left[\|g\|_{p}+\left\|\phi^{2} g^{\prime \prime}\right\|_{p}\right]+\left\|B_{n, r}(R(g, t, x), x)\right\|_{p}
\end{aligned}
$$

where $R(g, t, x)=\int_{x}^{t}(t-u) g^{\prime \prime}(u) d u$.

Now, we shall prove that

$$
\left\|B_{n, r}(R(g, t, x), x)\right\|_{p} \leq C n^{-1}\left\|\left(\phi^{2}+n^{-1}\right) g^{\prime \prime}\right\|_{p} .
$$

We prove this for $p=1$ and $p=\infty$. The cases $1<p<\infty$ follows again by Riesz-Thorin theorem.

Using (2.5) for the case $m=1$ and Lemma 2.4 , the case $p=\infty$ easily follows (see e.g. [5]).

For $p=1$, we derive (3.4) by applying Fubini's theorem twice, the definition of $H_{n}(u)$ and Lemma 2.5 as

$$
\begin{aligned}
\int_{0}^{\infty} \mid & B_{n, r}(R(g, t, x), x) \mid d x \\
\leq & \alpha(n, r) \int_{0}^{\infty} \sum_{k=0}^{\infty} p_{n+r, k}(x) \int_{0}^{\infty} b_{n-r, k+r}(t)\left|\int_{x}^{t}(t-u) g^{\prime \prime}(u)\right| d t d x \\
= & \alpha(n, r) \int_{0}^{\infty}\left|g^{\prime \prime}(u)\right|\left\{\int_{0}^{\infty} \int_{0}^{u}-\int_{0}^{u} \int_{0}^{\infty}\right\}(u-t) \\
& \times \sum_{k=0}^{\infty} p_{n+r, k}(x) b_{n-r, k+r}(t) d t d x d u \\
= & \alpha(n, r) \int_{0}^{\infty}\left|g^{\prime \prime}(u)\right| H_{n}(u) d u \\
\leq & C n^{-1}\left\|\phi^{2} g^{\prime \prime}\right\|_{1} \\
\leq & C n^{-1}\left\|\left(\phi^{2}+n^{-1}\right) g^{\prime \prime}\right\|_{1},
\end{aligned}
$$


where $C$ is independent of $n$. Hence (3.4) holds by Riesz-Thorin theorem for $1 \leq p \leq \infty$. Combining the estimates of (3.2), (3.3) and (3.4) we get

$$
\begin{aligned}
\left\|B_{n, r} f-f\right\|_{p}= & C\|f-g\|_{p}+C n^{-1}\left\{\|f-g\|_{p}+\|f\|_{p}+\left\|\phi^{2} g^{\prime \prime}\right\|_{p}\right. \\
& \left.+\left\|\left(\phi^{2}+n^{-1}\right) g^{\prime \prime}\right\|_{p}\right\} \\
\leq & C\left\{\|f-g\|_{p}+n^{-1}\left\|\phi^{2} g^{\prime \prime}\right\|_{p}+n^{-2}\left\|g^{\prime \prime}\right\|_{p}+n^{-1}\|f\|_{p}\right\} .
\end{aligned}
$$

Next taking the infimum over all $g \in \bar{W}_{p}^{2}(\phi,[0, \infty))$ on the right hand side, we get

$$
\left\|B_{n, r} f-f\right\|_{p} \leq C\left\{\bar{K}_{\phi}^{2}\left(f, n^{-1}\right)+n^{-1}\|f\|_{p}\right\}
$$

this completes the proof of Theorem 3.1.

Remark. The conclusion of Theorem 3.1 is true on the space $L_{p}[0, \infty), 1 \leq p<\infty$ (i.e. $\lim _{n \rightarrow \infty}\left\|B_{n, r} f-f\right\|_{p}=0$ for every $\left.f \in L_{p}[0, \infty)\right)$, since the most basic fact about $\omega_{\phi}^{2}\left(f, n^{-1}\right)$ is that

$$
\lim _{n \rightarrow \infty} \omega_{\phi}^{2}\left(f, n^{-1}\right)=0 \text { for all } f \in L_{p}[0, \infty), \quad 1 \leq p<\infty,
$$

or for all bounded functions $f \in C[0, \infty)$ which satisfy

$$
\lim _{x \rightarrow \infty} f(x)=L_{\infty}<\infty, \text { if } p=\infty(\text { cf. [2, p. 36]) }
$$

Acknowledgement. The author is grateful to the referee for many suggestions that grately improved this paper.

\section{References}

1. Z. Ditzian And K. Ivanov, Bernstein type operators and their derivatives, J. Approx. Theory 56 (1989), 72-90.

2. Z. Ditzian And V. Totik, "Moduli of smoothness," Springer Series in Computational Mathematics 9, Springer-Verlag, Berlin, Heidelberg, New York, 1987.

3. J. L. Durrmeyer, Une formule d'inversion, de la transformée de Laplace: Application á la Theorie des Moments, Thése de 3e Cycle, Faculté des Sciences de l'Universite de Paris, 1967.

4. V. Gupta, A note on modified Baskakov type operators, Approx. Theory and its Appl. 10(3) (1994), 74-78. 
5. M. Heilmann, Direct and converse results for operators of Baskakov-Durrmeyer type, Approx. Theory and its Appl. 5(1) (1989), 105-127.

6. A. SAHAi AND G. Prasad, On simultaneous approximation by modified Lupas operators, J. Approx. Theory 45 (1985), 122-128.

7. R. P. Sinha, P. N. Agrawal and V. Gupta, On simultaneous approximation by modified Baskakov operators, Bull. Soc. Math. Belg. Ser. B 42(2) (1991), 217-231.

\author{
Department of Mathematics \\ University of Roorkee \\ Roorkee-247667 (U.P.) \\ INDIA
}

Primera versió rebuda el 13 d'Octubre de 1994, darrera versió rebuda el 8 de Maig de 1995 